\title{
A double neon colour illusion
}

\author{
Rob van Lier \\ Nijmegen Institute for Cognition and Information (NICl), University of Nijmegen, PO Box 9104, \\ NL 6500 HE Nijmegen, The Netherlands; \\ e-mail: r.vanlier@nici.kun.nl; http://www.nici.kun.nl/People/LiervanRJ/index.html \\ Received 7 August 2000, in revised form 18 September 2001
}

\begin{abstract}
A new visual illusion is presented. When two neon-coloured illusory bands overlap each other, the filling-in at the region of overlap is perceived as different from those of the overlapping bands, resulting in an additional illusory shape at the region of overlap. An experiment with ten naïve participants was performed to measure the illusory percept. The results show that illusory surfaces may interact with each other, and, to a certain extent, create new illusory percepts.
\end{abstract}

\section{Introduction}

The perception of illusory shapes and colour is a well-studied topic in vision research. A striking example of both shape and colour illusion was provided by Varin (1971), who showed that the colour of certain physical inducers may spread throughout an illusory shape (Kanizsa 1976) - a phenomenon that also has been referred to as neon colour spreading (van Tuijl 1975). The resulting percept is characteristically one of a transparent coloured shape. As the assimilative colour caused by the coloured elements is perceptually detached from the background and transposed to a second surface, this process has also been referred to as perceptual scissioning (see, for example, Anderson 1997; Bressan et al 1997; Nakayama et al 1990; Spillmann and Dresp 1995). The perceptual phenomenon shown here demonstrates that such illusory surfaces may interact with each other, and, to a certain extent, create new illusory percepts. The displays consist of overlapping illusory neon-coloured bands that elicit a faint additional filling-in at the region of overlap.

\section{1 'Dark' neon colourings}

An example is shown in figure 1a. The display consists of four yellowish illusory bands, induced by the yellow segments within the white circular disks (see also http:// www.perceptionweb.com/perc0102/vanlier.html, display 1A). The area of the 'yellow' bands appears somewhat darker than its grey surroundings. On inspecting the regions where these bands overlap, an additional difference in lightness shows up. This region of overlap seems slightly darker-yellow than the rest of the bands. This phenomenon can be enhanced by introducing an apparent motion of the bands (cf Cicerone et al 1995). See Perception website for such a dynamic display (http://www.perceptionweb.com/ perc0102/vanlier.html, display 1B). Obviously, other colour settings may also be used to achieve the illusion (see Perception website for an additional static and dynamic example with a different colour setting: http://www.perceptionweb.com/perc0102/vanlier.html, displays $2 \mathrm{~A}$ and $2 \mathrm{~B}$ ).

\section{2 'Light' neon colourings}

In the previous example, the bands appear darker than the surroundings. It is well known that other configurations may comprise neon-coloured shapes that appear lighter than the background (see, eg, Bressan et al 1997; van Tuijl and de Weert 1979; Varin 1971). Such light neons can be achieved, for example, when using black disks, deep-blue segments, and 


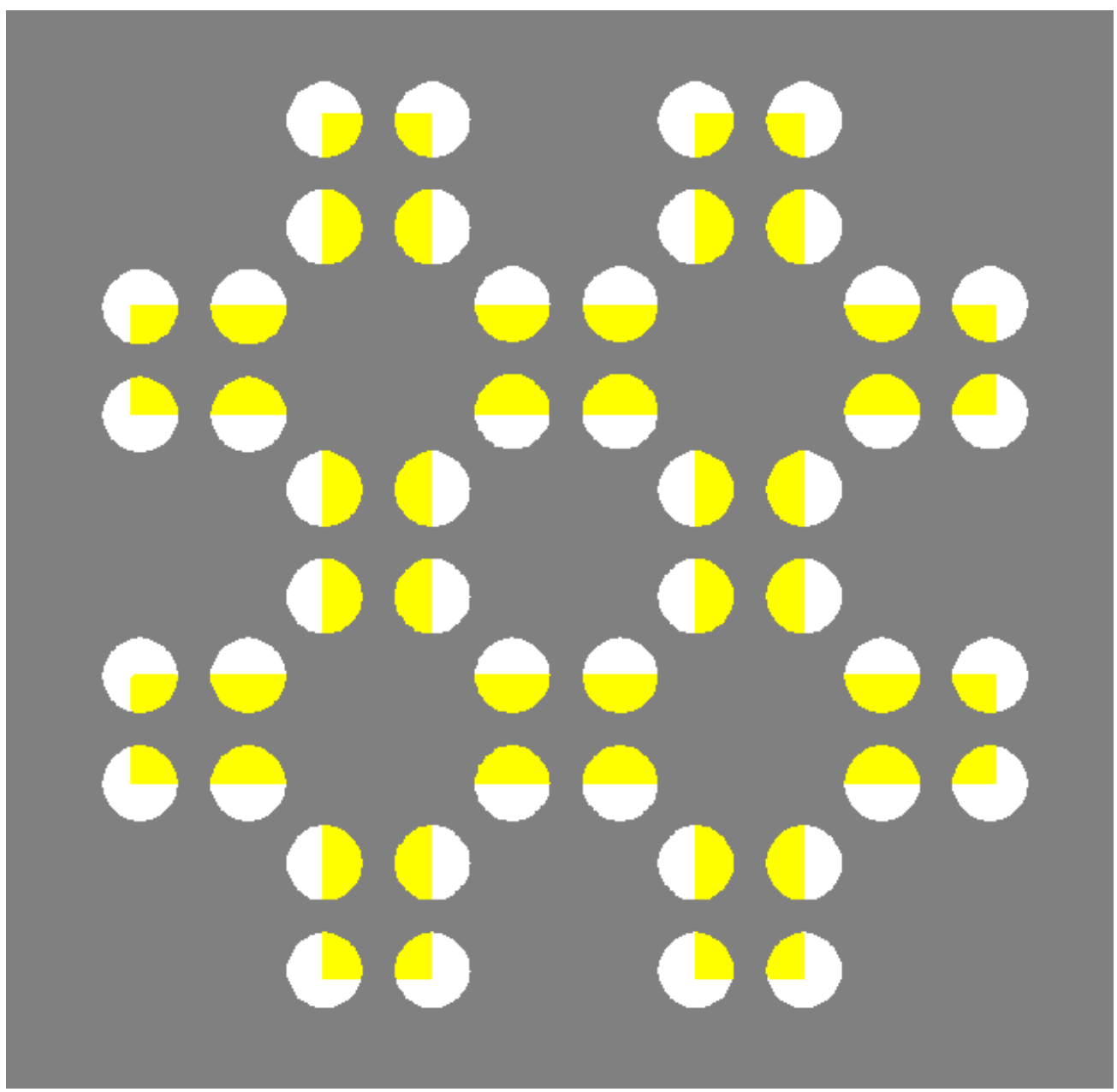

(a)

Figure 1. Two coloured examples of displays with overlapping neon bands. Each display consists of four overlapping neon-coloured bands. The perceived lightness at the regions of overlap appears to be different from the lightness of the overlapping bands. The overlapping yellow neon bands (a) result in a darker overlapping region whereas overlapping blue neon bands (b) result in a lighter overlapping region. At the Perception website (http://www.perceptionweb.com/perc0102/ vanlier.html) these and various other colour examples can be seen, see text for details.

a white background (see figure 1b or http://www.perceptionweb.com/perc0102/vanlier.html, display 3A). On inspecting this configuration, it appears that the regions of overlap are perceived to be slightly lighter than the bands themselves. Again, this effect can be enhanced in a dynamic display (see http://www.perceptionweb.com/perc0102/vanlier.html, display 3B).

\section{Experiment}

An experiment was run to test the difference in lightness at the region of overlap for naïve observers.

\subsection{Participants}

Ten undergraduate students participated in the experiment. All participants were naïve with respect to the goal of the experiment. The participants either received course credit or were paid an equivalent of about $\$ 5$. 


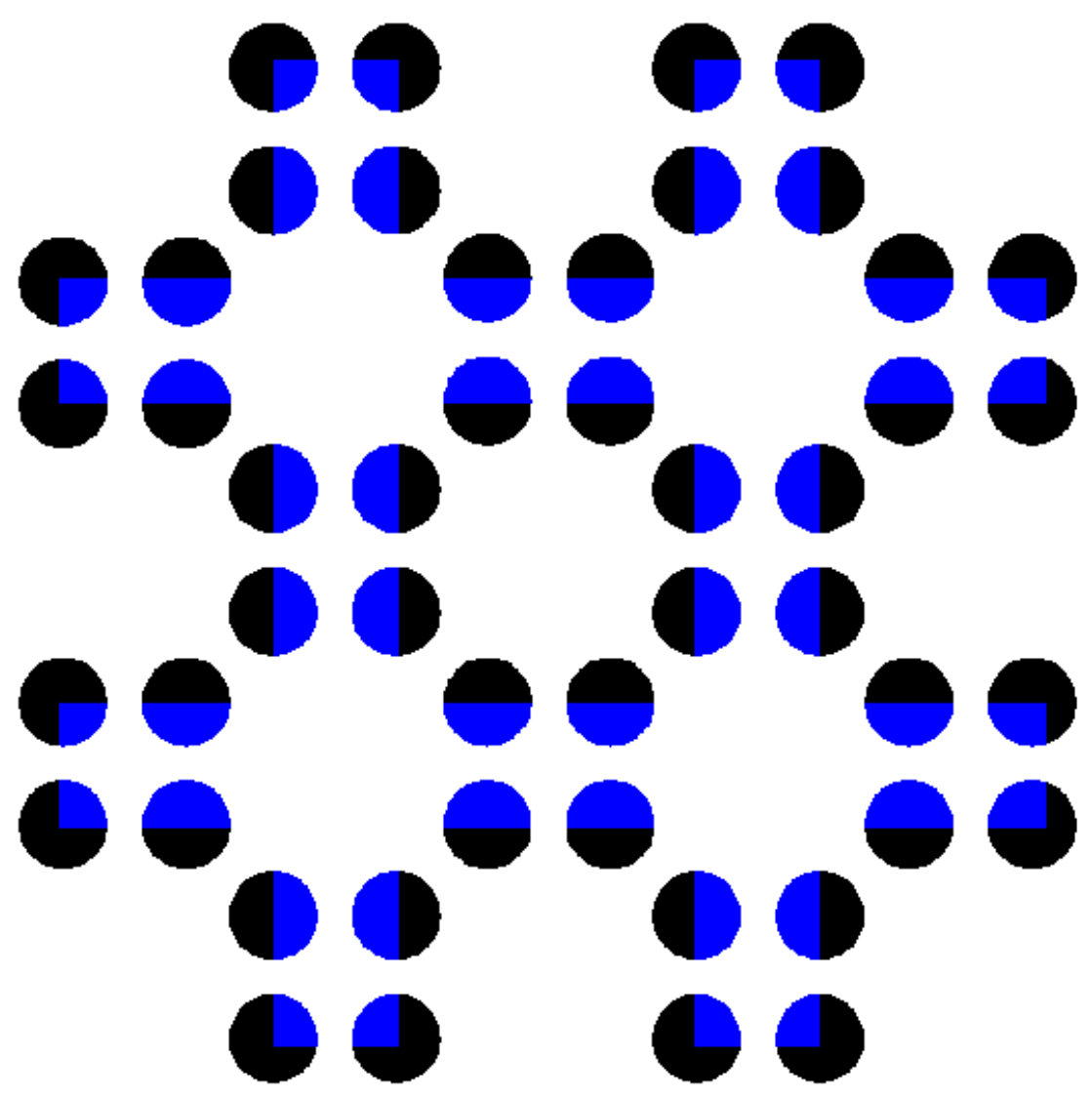

(b)

Figure 1 (continued)

\subsection{Apparatus}

The experiment was carried out on a PC with a Pentium-II processor and a Philips Brilliance 109 monitor. The experiment was run in Superlab (Cedrus Inc.) environment.

\subsection{Stimuli and procedure}

In the experiment, each pattern always consisted of just two overlapping neon bands (see figure 2a). In this arrangement, there were four different positions of the neon bands (top-left, top-right, bottom-left, and bottom-right). Two different types of neon patterns were used: one with a dark neon colouring (grey background, white disks, yellow inducers as in figure 1a), referred to as type I patterns, and one with a light neon colouring (white background, black disks, blue inducers as in figure 1b), referred to as type II patterns.

Within a trial, the following events occurred. First, the pattern appeared on the screen. After $1500 \mathrm{~ms}$, two small, grey markers with square outlines were positioned on the pattern. The participants were asked to judge which marker was placed on the lightest background. In figure $2 b$, all possible positions of the markers are shown. Three different fields in which the markers could be positioned can be distinguished: 


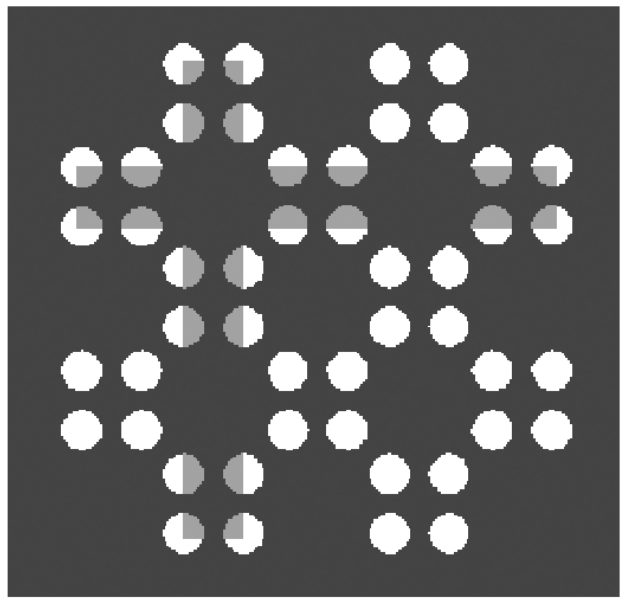

(a)

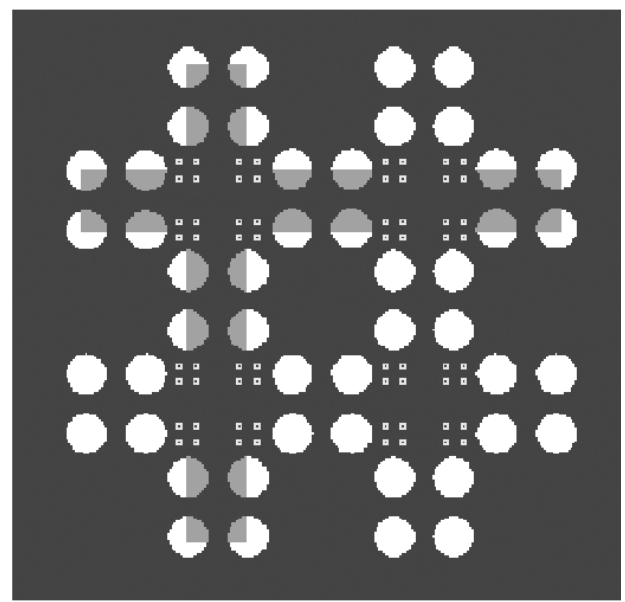

(b)

Figure 2. (a) The stimuli in the experiment are derived from the patterns as shown in figure 1 . Each stimulus consists of two overlapping bands. (b) All possible positions of the markers (small square outlines).

non-neon field (marker outside neon band), single neon field (marker within a neon band), and double neon field (marker within region of overlapping neon bands). In the following, these fields will be indicated with the abbreviations $\mathrm{N}, \mathrm{S}$, and $\mathrm{D}$, respectively. Markers relatively close to each other (within each quadruplet) were compared with each other. All comparisons were made for each of the four positions of the neon bands. As the number of critical SD comparisons (one marker in an S field and the other in a D field) was relatively low, these comparisons were presented twice. In total, the experimental design led to 832 stimuli per participant, presented in a random order. Responses were given by pressing arrow keys on a PC keyboard such that the arrow pointed toward the marker on the judged lightest field. A response 'equal lightness' could be given as well (by pressing a key marked 'same').

\section{Results}

Clearly, for the present purpose our main interest lies in the SD comparisons. However, these results should be compared with control conditions in which markers are placed at the same locations but without neon field differences (with both markers either in a single neon field or in a non-neon field). In addition to this, the results of SD comparisons are compared with the results of NS comparisons. We have restricted ourselves to horizontal and vertical comparisons, as only for these directions there were SD comparisons to be made.

Table 1 shows the proportions of the three possible lightness judgments $\left(L^{+}, L^{=}\right.$, $L^{-}$) for the marker in the critical field (D or S, for SD or NS comparisons, respectively) relative to the marker in the reference field ( $\mathrm{S}$ or N, for SD or NS comparisons, respectively). For type I patterns, $L^{-}$judgments (darker) prevail for both SD and NS comparisons. For type II patterns, $L^{+}$judgments (lighter) prevail, again for both SD and NS comparisons. For the control comparisons of both displays, large proportions of $L^{=}$judgments (equal lightness) are prevalent. To test the overall effect of the neon bands on the judgments, we have calculated for each participant $\Delta L=L^{+}-L^{-}$for both the experimental and control comparisons and then compared them by means of $t$-tests. These $t$-tests revealed significant values for all comparisons (type I pattern/ SD: $t_{9}=6.010, p<0.001$; type I pattern/NS: $t_{9}=8.112, p<0.001$; type II pattern/SD: $t_{9}=2.703, p<0.05$; type II pattern/NS: $t_{9}=2.487, p<0.05$ ). The graph in figure 3 
Table 1. The proportion of lightness judgments for the marker in the critical field (D or S, for SD or NS comparisons, respectively) relative to the marker in the reference field ( $\mathrm{S}$ or $\mathrm{N}$, for SD or NS comparisons, respectively). SD, single-neon/double-neon comparison; NS, nonneon/single-neon comparison; $L^{+}$, marker in critical field judged to be on a lighter background than marker in reference field; $L^{=}$, marker in critical field judged to be on a background with same lightness as marker in reference field; $L^{-}$, marker in critical field judged to be on a darker background than marker in reference field. Type I: 'dark'-yellow neon bands (white disks, yellow inducers, grey background); type II: 'light'-blue neon bands (black disks, blue inducers, white background).

\begin{tabular}{|c|c|c|c|c|c|c|}
\hline & \multicolumn{3}{|c|}{ SD comparison } & \multicolumn{3}{|c|}{ NS comparison } \\
\hline & $L^{+}$ & $L^{=}$ & $L^{-}$ & $L^{+}$ & $L^{=}$ & $L^{-}$ \\
\hline \multicolumn{7}{|l|}{ Type I } \\
\hline experiment & 0.02 & 0.30 & 0.68 & 0.03 & 0.13 & 0.84 \\
\hline control & 0.04 & 0.90 & 0.06 & 0.03 & 0.91 & 0.06 \\
\hline \multicolumn{7}{|l|}{ Type II } \\
\hline experiment & 0.63 & 0.23 & 0.14 & 0.68 & 0.12 & 0.20 \\
\hline control & 0.05 & 0.91 & 0.04 & 0.04 & 0.93 & 0.03 \\
\hline
\end{tabular}

shows the corrected judged lightness, $\Delta L^{\prime}$. These $\Delta L^{\prime}$ values have been obtained by taking the difference between the $\Delta L$ values of the experimental conditions and the $\Delta L$ values of the corresponding control conditions. A positive $\Delta L^{\prime}$ value means a (control-corrected) 'lighter' judgment for markers in a critical field, whereas a negative value indicates a 'darker' judgment for those positions. Whereas the individual scores generally follow overall judgments, a rather strange exception occurs for participant 4 who has judged the neon fields of the type II patterns to be darker for both the SD and NS comparisons. Another exception can be seen for participant 7 who apparently did not see any lightness differences at all. Participant 5 responded with almost no
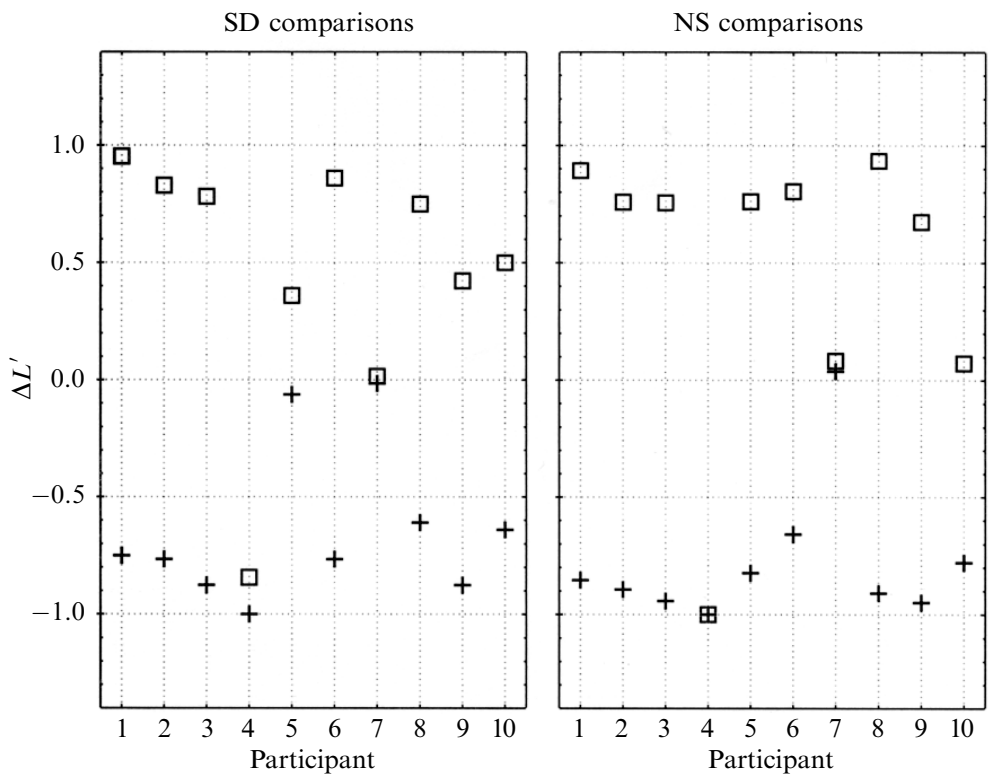

+ type I (yellow)
$\square$ type II (blue)

Figure 3. Graph of the individual data (participants 1-10). The vertical axis shows the corrected judged lightness, $\Delta L^{\prime}$. A positive value of $\Delta L^{\prime}$ means a (control-corrected) 'lighter' judgment for markers in a critical field, whereas a negative value indicates a 'darker' judgment for those positions. 
lightness difference for the SD comparisons of type I patterns, whereas she appeared to perceive them for the NS comparisons, and participant 10 responded with almost no lightness difference for the NS comparisons on type II patterns, whereas she responded with a lightness difference for the corresponding SD comparisons in about half of the cases. On the whole, however, the $\Delta L^{\prime}$ values of SD and NS comparisons appear not to be very different from each other [a $t$-test comparing these values revealed no significant difference $\left(t_{19}=1.186, p=0.250\right)$, whereas a correlational analysis revealed a highly significant value $\left.\left(r^{2}=0.91, p<0.0001\right)\right]$.

\section{Discussion}

The experiment has confirmed the perception of a different lightness when two neon bands cross each other. More specifically, it has been shown that crossing dark neon bands may elicit a darker overlap and crossing light neon bands may elicit a lighter overlap. These results suggest an additive-like process of the illusory lightnesses. However, further research on overlapping neon-coloured shapes should teach us more about the precise properties of overlapping neon bands. For example, preliminary observations in our laboratory on displays with overlapping dark versus light neon bands show that such a dark-light overlap reveals a region the lightness of which lies in between the lightness appearances of the overlapping bars (see http://www.perceptionweb.com/perc0102/ vanlier.html, display 4A for a static version and display 4B for a dynamic version). Future research may also relate the lightness appearances of overlapping regions to the impression of transparency; if for the displays presented here we rely on the judged lightness, then all overlapping regions seem to agree with a so-called bistable transparency (Adelson and Anandan 1990; cf Anderson 1997). In connection with this, the possibility of perceived hue differences might be examined as well (see Perception website for an example of crossing yellow and blue neon bands; http://www.perceptionweb.com/perc0102/vanlier.html, displays $5 \mathrm{~A}$ and $5 \mathrm{~B})$.

Although quite a number of studies have been devoted to topics such as illusory contours and colour filling-in, it is still not clear how the visual system precisely creates these percepts (eg see reviews by Bressan et al 1997; Pessoa et al 1998; Spillmann and Dresp 1995). The present phenomenon is in line with previous findings that show a close relation between neon-colour filling-in and illusory contours (eg Takeichi et al 1992; Watanabe and Takeichi 1990). In addition, it is known that the perceptual strength of neon colouring depends on various factors such as colour, luminance, shape, or motion (eg Cicerone et al 1995; Redies and Spillmann 1981; van Tuijl and Leeuwenberg 1979; van Tuijl and de Weert 1979; de Weert and van Kruysbergen 1987). Given the present results, it seems reasonable to presume that factors that enhance single neon colour illusions also enhance double neon colour illusions (the dynamic displays can be considered as an illustration of this), but future research may reveal whether this is always the case.

So far, the present phenomenon seems to need the physically induced illusory percepts as a prerequisite and can therefore perhaps best be characterised as a secondorder illusion. The fact that not direct physical input but something intermediate seems to serve as the major triggering of this filling-in phenomenon only contributes to the subtlety of this intriguing illusion.

Acknowledgements. This article was written while I received a grant from the Royal Netherlands Academy of Arts and Sciences (KNAW). I thank Charles de Weert for fruitful discussions and all of his suggestions, and also Paola Bressan and an anonymous reviewer for their helpful comments on a previous version of this article. 


\section{References}

Adelson E H, Anandan P, 1990 "Ordinal characteristics of transparency" paper presented at the AAAI-90 Workshop on Qualitative Vision, July 29, 1990, Boston, MA

Anderson B L, 1997 "A theory of illusory lightness and transparency in monocular and binocular images: the role of contour junctions" Perception $26419-453$

Bressan P, Mingolla E, Spillmann L, Watanabe T, 1997 "Neon color spreading: a review" Perception $261353-1366$

Cicerone C M, Hoffman D D, Gowdy P D, Kim J S, 1995 "The perception of color from motion" Perception \& Psychophysics $57761-777$

Kanizsa G, 1976 "Subjective contours" Scientific American 234 48-52

Nakayama K, Shimojo S, Ramachandran V S, 1990 "Transparency: relation to depth, subjective contours, luminance, and neon color spreading" Perception 19 497-513

Pessoa L, Thomson E, Noë A, 1998 "Finding out about filling-in: A guide to perceptual completion for visual science and the philosophy of perception" Behavioral and Brain Sciences 21 723-802

Redies C, Spillmann L, 1981 "The neon color effect in the Ehrenstein illusion" Perception 10 $667-681$

Spillmann L, Dresp B, 1995 "Phenomena of illusory form: can we bridge the gap between levels of explanation" Perception $241333-1364$

Takeichi H, Shimojo S, Watanabe T, 1992 "Neon flank and illusory contour: interaction between the two processes leads to color filling-in" Perception $21313-324$

Tuijl H F J M van, 1975 "A new visual illusion: Neonlike color spreading and complementary color induction between subjective contours" Acta Psychologica 39441 - 445

Tuijl H F J M van, Leeuwenberg E L J, 1979 "Neon color spreading and structural information measures" Perception \& Psychophysics $25269-284$

Tuijl H F J M van, Weert C M M de, 1979 "Sensory conditions for the occurrence of the neonspreading illusion" Perception $8211-215$

Varin D, 1971 "Fenomeni di contrasto e diffusione cromatica nell'organizzazione spaziale del campo percettivo" Rivista di Psicologia $65101-128$

Watanabe T, Takeichi H, 1990 "The relation between color spreading and illusory contours" Perception \& Psychophysics $47457-467$

Weert C M M de, Kruysbergen A W H van, 1987 "Subjective contour strength and perceptual superimposition: transparency as a special case", in The Perception of Illusory Contours Eds S Petry, G E Meyer (New York: Springer) pp 165-170 


\section{APPENDIX}

Material on the website (http://www.perceptionweb.com/perc0102/vanlier.html)

Display 1. (A) Static display of overlapping neon-coloured illusory bands (white disks, yellow segments, grey background). The regions of overlap appear to be filled-in with a darker yellow, evoking a transparency impression at the illusory crossing. (B) Dynamic version of display A. In this display two bands make small oscillatory movements (whereas in fact only the position and shape of the yellow segments within the surrounding disks change).

Display 2. (A) Another example of a static version of overlap with dark neon-coloured bands (white disks, red background, pale-blue segments). (B) Dynamic version of display A. Display 3. (A) Static configuration with light neon-coloured bands (black disks, deepblue segments, white background). The regions of overlap appear lighter than the bands themselves. (B) Dynamic version of display A.

Display 4. (A) Static configuration of overlapping dark neon-coloured bands and light neon-coloured bands (white disks and pale-blue segments, black disks and deep-blue segments, grey background). (B) Dynamic version of display A. Notice that, in the mixed lightness crossings in this display, the lightness of the moving band seems to prevail.

Display 5. (A) Static version of a multicolour display (white disks, yellow and pale-blue segments, grey background). The display leaves the impression that there is darker yellow at the yellow-yellow overlap, a darker blue at the blue-blue overlap, whereas there is an indefinite colour at the yellow-blue crossings. At the latter crossings, a rivalry between the bands can also be experienced. (B) Dynamic version of display A. Notice that, in the mixed colour crossings in this display, the colour of the moving band seems to prevail. 